\title{
A model for fibre contact in planar random fibre networks
}

\author{
W. W. SAMPSON \\ Department of Textiles and Paper, UMIST, P.O. Box 88, Manchester, M60 10D, UK \\ E-mail:w.sampson@umist.ac.uk
}

\begin{abstract}
A model is presented for the expected degree of contact between fibres in isotropic near-planar random fibre networks. The statistics of fibre contact in two-dimensional fibre networks are considered and the expressions derived are developed to allow the fractional contact area in structures formed by the superposition of two-dimensional structures to be derived. These expressions allow the fractional contact area to be expressed in terms of the network porosity only. For thin networks, the fractional contact area may be expressed in terms of network porosity and the expected coverage of the network. Theory permitting the determination of the expected area of one contact and hence the expected number of contacts per fibre is presented also. Good agreement is found between the expressions derived for the fractional contact area and data from the literature. (c) 2004 Kluwer Academic Publishers
\end{abstract}

\section{Introduction}

The statistical geometry of random fibrous and line networks has been studied by many workers. Miles [1] considered the statistics of polygons formed by the intersections of a system of straight lines positioned according to a point Poisson process in two dimensions and with uniformly distributed orientation to a given direction. Miles showed that the expected number of sides per polygon in such a network is 4 and that distribution of the radii of circles inscribed within the polygons is negative exponential. These results were confirmed in the Monte-Carlo study of Piekaar and Clarenburg [2] and were developed by Corte and Lloyd [3] and Dodson and Sampson [4] who derived the pore radius distribution for random and clumped stochastic line networks respectively.

Industrially manufactured stochastic fibrous networks include nonwoven fabrics, fibrous filter media and paper. Naturally, the fibres used in the manufacture of such materials have finite length and width and as such these have been incorporated in statistical models. Kallmes and Corte [5] considered the statistical geometry of 'two-dimensional' random fibre networks, and defined a random fibre network as one where fibre centres are positioned according to a point Poisson process in two dimensions and the orientation of fibre axes to a given direction has a uniform distribution. The number of fibres covering a point is termed the coverage and Kallmes and Corte defined two-dimensional networks as those where less than $1 \%$ of the area has coverage greater than two. They derived expressions for several structural features including the expected number of crossings per fibre, the expected distance between such crossings and the expected pore area. The distribution of mass in random fibre networks was derived by Dodson
[6] and was shown to be a function of the scale of inspection, the mean mass per unit area of the network and the length, width and mass per unit length of the constituent fibres.

An additional feature of interest in the structure of stochastic fibrous networks is the amount of contact that occurs between fibres. This property is important since it determines the available area for inter-fibre bonding and hence contributes strongly to many of the mechanical properties of the network. In an industrial context, the mechanical deformation of fibrous webs such as paper, nonwoven fabrics and fibrous filters under strain, and ultimately their failure, are important in their manufacture and in converting and end-use applications. In the cases of paper and paperboard inparticular, these are typically manufactured to a tensile strength dependent on end-use application and specified by the customer; the preparation of fibres for manufacture of the web primarily involves developing their potential for bonding to meet these specifications.

Deng and Dodson [7], following Kallmes et al. [8], considered a two-dimensional network of expected coverage $\bar{c} \leq 1$, such that less than $2 \%$ of the area is covered by more than three fibres. They showed that the fraction of fibre surface in contact with other fibres, termed the Relative Bonded Area (RBA) was given by

$$
\mathrm{RBA}=1-\frac{1-e^{-\bar{c}}}{\bar{c}},
$$

where $\bar{c}$ is the expected coverage of the network.

The maximum out-of-plane dimension of web or sheet-like stochastic fibrous networks is typically only a fraction of the expected length of the fibres from which they are made. Radvan, Dodson and Skold 
[9], in an elegant series of experiments showed that the structure of paper is essentially layered with fibre axes oriented within only a few degrees of the network plane. Whilst no discrete layers exist within conventionally manufactured stochastic fibre networks, the experiments of Radvan, Dodson and Skold indicate that fibres lie one on top of another and are not intertwined to any significant extent. Accordingly, several workers have modelled these structures as multi-layer composite structures of two-dimensional networks. Soszyński [10] used the binomial distribution to model the expected amount of contact between fibres in a structure of $n$ layers with solid fraction $(1-\varepsilon)$ such that

$$
\mathrm{RBA}=\frac{n}{n-1}(1-\varepsilon)^{2},
$$

and defined number of layers in a given structure in terms of the thicknesses of the fibres and the network.

Measurements of the relative bonded area made on micrographs of cross-sections of laboratory formed networks have been recently presented by Niskanen and Rajatora [11]. They found good agreement between their data and the expression,

$$
\mathrm{RBA}=\frac{a}{\bar{h}}\left(1-\frac{1-e^{-\bar{c}}}{\bar{c}}\right)
$$

where $a$ is the resolution of their measuring system and $\bar{h}$ is the measured mean vertical separation of fibres. We note the similarity between Equations 1 and 3 and that Niskanen and Rajatora found good agreement between Equation 3 and computer simulations of high coverage networks [12].

Gates and Westcott [13] derived expressions for the upper and lower bounds of fibre contact areas incorporating the thickness of fibres, $t$ as a variable controlling fibre flexing. They presented also the approximation,

$$
\mathrm{RBA} \approx \begin{cases}\bar{c} e^{-\bar{c} t / 2} & \text { for } \bar{c} \leq 2 / t \\ \frac{2}{e t} & \text { for } \bar{c} \geq 2 / t\end{cases}
$$

and found good agreement with data from simulations. Note that the simulations of Hellén et al. [12] incorporate the flexing of fibres as an input parameter also.

Here we apply the concept of multiplanar fibrous networks to the study of the fractional contact area in random fibre networks. The expressions derived allow the fractional contact area to be expressed in terms of the network density only for thick networks, and in terms of the network density and mean coverage for thin networks. The expressions derived are compared with data from the literature. From the functions giving the fractional contact area, we proceed to determine the expected area of a single contact and hence the expected number of contacts per fibre.

\section{Theory}

We consider first the statistical geometry of twodimensional random fibre networks and derive expres- sions for the fraction of the surface of a chosen fibre that is in contact with other fibres. We proceed to consider the superposition of two-dimensional networks to form multiplanar structures more representative of typical commercially formed networks. We define a random network of fibres as one where the fibre centres are positioned according to a point Poisson process in two dimensions and the orientation of fibre axes to a given direction has a uniform distribution. The number of fibres covering a point is termed the coverage and the Poisson probability that a given point has coverage, $c$ is given by

$$
P(c)=\frac{\bar{c}^{c} e^{-\bar{c}}}{c !}
$$

where $\bar{c}$ is the mean coverage of points in the network.

\subsection{Two dimensions}

Following Sampson [14], we define the mean coverage of a two-dimensional network in terms of its fractional open area, $\varepsilon$ such that

$$
\varepsilon=e^{-\bar{c}}
$$

and therefore,

$$
\bar{c}=\log (1 / \varepsilon) .
$$

Sampson showed that for such a two-dimensional random fibre network, the expected pore radius was real and positive for $e^{-\frac{\pi}{2}} \leq \varepsilon<1$, this corresponding to a networks of mean network coverage, $\bar{c} \leq \pi / 2$. Above this mean coverage, the theory of Sampson [14] yields a negative value for the mean pore radius of a twodimensional network. Naturally, the mean pore radius of a fibre network can be zero only when the porosity of the network is zero also; the upper limit on mean coverage arises because the model is unable to distinguish between voids that are closed by the edges of fibres being adjacent to each other, and those that are closed when the edges of fibres overlap. In the sequel, we bear this in mind in our discussion of the applicable range of the theory developed here. Accordingly, we define our two-dimensional network as one of mean coverage less than $\pi / 2$ or equivalently, of mean porosity greater than $e^{-\frac{\pi}{2}} \approx 0.208$ and examine the behaviour of the expressions derived for all porosities above this mathematically permissible limit.

Substitution of Equation 6 in Equation 5 gives,

$$
P(c)=\frac{\varepsilon}{c !} \log (1 / \varepsilon)^{\mathrm{c}} .
$$

Consider now any given fibre within such a twodimensional network; Fig. 1 shows the contact states of fibres in regions of coverage between 1 and 5 . In regions of coverage 1 , the fibre is in contact with no others; where the coverage is 2 , any fibre is in contact with the other fibres either above or below it, so the expected number of contacts $\bar{\phi}$ is 1 . Where the coverage at points is 3 , there are three ways to chose a fibre: two 


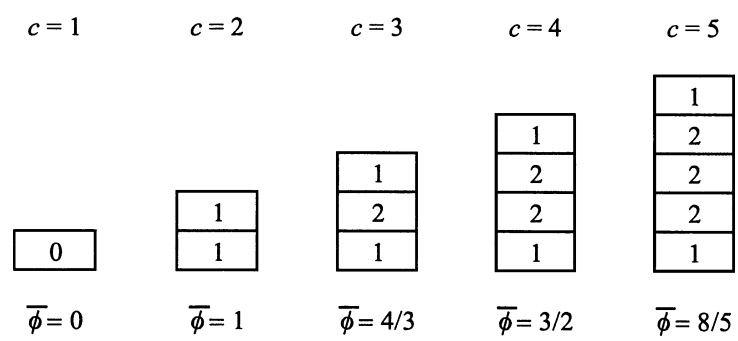

Figure 1 Contact states of fibres in regions of coverage between 1 and 5 . Each rectangle represents a fibre and the number within it represents the number of contacts that it makes. The value of $\bar{\phi}$ beneath each coverage group represents the expected number of contacts in regions with that coverage.

where the chosen fibre is in contact with others on one side only and one where it is in contact on both sides, so we have $\bar{\phi}=(1+2+1) / 3=4 / 3$. In the general case, in regions of coverage $c$ there are 2 ways of choosing a fibre that is in contact with one other, and $(c-2)$ ways that it is contact on both sides. Thus, the expected number of contacts at points with coverage $c$ is,

$$
\bar{\phi}(c)=\frac{2(c-1)}{c} .
$$

The expected fraction of any given fibre in a twodimensional network that is in contact with other fibres is given by

$$
\Phi_{2 \mathrm{D}}=\frac{1}{\bar{c}} \sum_{c=1}^{\infty} \bar{\phi}(c) P(c),
$$

We have defined our two-dimensional network as one with mean coverage up to $\pi / 2$. For such a network, the probability that the coverage is greater than 5 is less than $1 \%$. Considering regions of coverage no greater than 5 gives therefore

$$
\begin{aligned}
\Phi_{2 \mathrm{D}} \approx & \varepsilon \log (\varepsilon)\left(-\frac{1}{2}+\frac{2}{9} \log (\varepsilon)-\frac{1}{16} \log (\varepsilon)^{2}\right. \\
& \left.+\frac{1}{75} \log (\varepsilon)^{3}+\cdots\right) .
\end{aligned}
$$

\subsection{Multiplanar structures}

Consider now a structure formed by the superposition of two-dimensional networks with independent and identical distributions of contacts as described above. Assuming that each two-dimensional network has some small but finite thickness perpendicular to its plane, we consider the space not occupied by fibres to have volume where in the two-dimensional case it had only area; as such, we consider the fractional open area and the fraction void volume or porosity to be equivalent.

Each layer of such a multiplanar network is assumed to make new contacts with the layers immediately above and below it only; accordingly we consider first a three layered structure. A given fibre in the central layer may form additional contacts with the surrounding layers in those regions where its own layer has no contact on either side, or is in contact with other fibres above or below it only. The fraction of new contacts occurring between layers is $(1-\varepsilon)^{2}$; of this fraction, additional contact is made with all regions in the central layer with coverage $1 ; 1 / 2$ the regions with coverage $2 ; 1 / 3$ of regions with coverage 3 , and in general $1 / c$ of all regions of coverage $c$. The additional fraction of a given fibre in the central layer that contacts fibres from adjacent layers is therefore given by

$$
\Phi^{*}=2(1-\varepsilon)^{2} \sum_{c=1}^{\infty} \frac{1}{c} P(c)
$$

where the coefficient 2 is included to account for contacts generated on both sides of the central layer. Again truncating the sum to consider regions of coverage up to 5 only, this yields,

$$
\begin{gathered}
\Phi^{*} \approx 2 \varepsilon(1-\varepsilon)^{2} \log (\varepsilon)\left(-1+\frac{\log (\varepsilon)}{4}-\frac{\log (\varepsilon)^{2}}{18}\right. \\
\left.+\frac{\log (\varepsilon)^{3}}{96}-\frac{\log (\varepsilon)^{4}}{600}+\cdots\right)
\end{gathered}
$$

Thus, the expected fraction of a fibre in the body of the network that is in contact with other fibres is given by

$$
\Phi_{\mathrm{mp}}=\Phi_{2 \mathrm{D}}+\Phi^{*} .
$$

Equation 13 is plotted against porosity in Fig. 2. The expressions of Deng and Dodson [7] and Soszyński [10], as given by Equations 1 and 2 with $n=\infty$, respectively are plotted in Fig. 2 also. We observe that Equation 13 predicts a higher fractional contact area at a given density than the theory of Soszyński and that when $\varepsilon=e^{-\pi / 2}, \Phi_{\mathrm{mp}}=0.992$ such that fibres in the network are approaching full contact. Soszyński's equation, on the other hand, gives $\Phi_{\mathrm{mp}} \rightarrow 1$ as $\varepsilon \rightarrow 0$; this is discussed further in the sequel. The equation of Deng and Dodson is strictly applicable to thin networks only and gives a similar estimate of the fractional contact area as Equation 13 for porosities above about 0.8 .

\subsubsection{Surface effects}

Towards the top and bottom surfaces of a multiplanar network, the probability of contact with other fibres

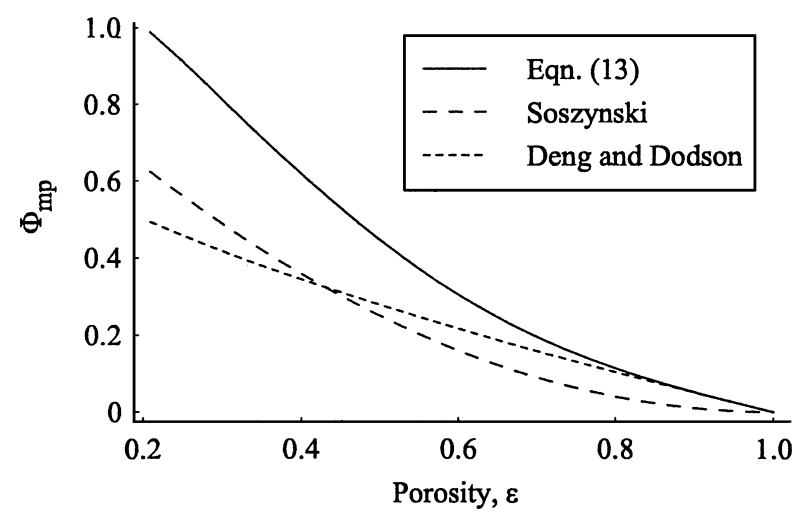

Figure 2 Influence of porosity on fractional contact area of a fibre in the bulk of multiplanar network. 
decreases. The number of two-dimensional layers that are required to form a multiplanar network of given mean coverage $\bar{c}_{\text {mp }}$ may be determined as

$$
n=\frac{\bar{c}_{\mathrm{mp}}}{\bar{c}}=\frac{\bar{c}_{\mathrm{mp}}}{\log (1 / \varepsilon)}
$$

Thus, the porosity of the network defines the coverage of a layer and hence the number of layers required to model a given multiplanar structure; the mean coverage of the network is independent of its porosity however.

Commercially formed fibre networks are typically manufactured to a specified mass per unit area or areal density. For a network of mean areal density $\bar{\beta}$ formed from fibres of width, $\omega$ and mass per unit length $\delta$ the mean coverage is $\bar{\beta} \omega / \delta$ and hence the number of layers, $n$ is given by,

$$
n=\frac{\beta \omega}{\delta \log (1 / \varepsilon)}
$$

For such a structure the expected fraction of any fibre that is in contact with other fibres is,

$$
\Phi_{\mathrm{mp}}^{*}=\frac{1}{n}\left((n-2) \Phi_{\mathrm{mp}}+\Phi^{*}\right)
$$

Equation 16 is plotted in Fig. 3 for the range of porosity permitted by the model; the broken lines represent the fractional contact area neglecting the contribution of surfaces as given by Equation 13 and the dotted line represents the coverage at which Equation 16 given 95\% of the fractional contact area predicted by Equation 13. This dotted line therefore provides an indication of the contribution of the fibres at the surface of the network to the global average fractional contact area. We observe that this contribution is greater for dense networks, i.e., those with low porosity, than for networks with more open structures.

The $95 \%$ threshold guides the classification of 'thin' or 'thick' networks in terms of their porosity and mean coverage. For example, a network of porosity 0.5 may be considered thick enough to estimate the fractional

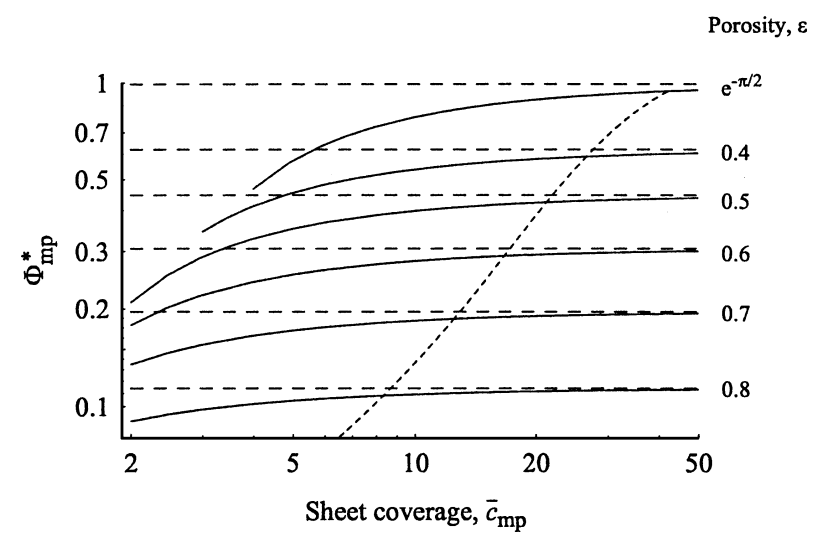

Figure 3 Influence of coverage on fractional contact area for structures of different porosity. The broken horizontal lines represent the fractional contact area neglecting the contribution of surfaces as given by Equation 13 and the dotted line represents the coverage at which Equation 16 gives $95 \%$ of the fractional contact area predicted by Equation 13 . contact area neglecting the contribution of surfaces using Equation 13 if it has mean coverage greater than about 20 , whereas a network of porosity 0.8 may be considered thick enough to apply Equation 13 if it has mean coverage greater than about 8 .

\subsection{Number of contacts per fibre}

Consider now a thin random network of fibres of length $\lambda$ and width $\omega$. When the mean coverage of the network $\bar{c}$ is less than 0.436 , the fraction of the area with coverage greater than 2 is less than $1 \%$. The expected number of fibre crossings per fibre in such a network is given by Kallmes and Corte [5] as,

$$
\bar{n}_{2 \mathrm{D}}=\frac{2 \bar{c} \lambda}{\pi \omega} \quad \text { for } \quad \bar{c} \leq 0.436
$$

For such a thin network, the fractional contact area is given by $\Phi_{2 \mathrm{D}}$ and the expected area of a given fibre that is in contact with others is given by

$$
\bar{A}_{\mathrm{c}}=2 \lambda \omega \Phi_{2 \mathrm{D}}
$$

such that the expected area of a single contact is

$$
\begin{aligned}
\bar{A}_{1} & =\frac{\bar{A}_{\mathrm{c}}}{\bar{n}_{2 \mathrm{D}}}, \\
& =\frac{\pi \omega^{2}}{\bar{c}} \Phi_{2 \mathrm{D}}
\end{aligned}
$$

It follows directly from Equation 17 that when the expected number of crossings per fibre is 1 , the expected coverage is $\bar{c}=\pi \omega /(2 \lambda)$ such that $\varepsilon=e^{\frac{-\pi \omega}{2 \lambda}}$. Denoting the fractional contact area of a two dimensional network with this coverage, $\Phi_{2 \mathrm{D}}^{\text {crit }}$ we have therefore,

$$
\bar{A}_{1}=2 \lambda \omega \Phi_{2 \mathrm{D}}^{\mathrm{crit}}
$$

For infinitely long fibres every crossing will be a parallelogram of area $\omega^{2} / \sin (\theta)$ where $\theta$ is the angle at which a pair of fibres cross. It is readily shown that the expected value of $\sin (\theta)$ in a network of fibres with a uniform distribution of orientations is $2 / \pi$, so the expected area of a crossing in a network of infinitely long fibres is $\pi \omega^{2} / 2$. Substitution of $\Phi_{2 \mathrm{D}}$ from Equation 10 when $\varepsilon=e^{-\frac{\pi \omega}{2 \lambda}}$ into Equation 21 and taking the limit as the fibre length tends to infinity yields,

$$
\lim _{\lambda \rightarrow \infty}\left(\bar{A}_{1}\right)=\frac{\pi}{2} \omega^{2}
$$

recovering the established result for infinitely long fibres. As this treatment is dependent on the theory presented for the fractional contact area of a two dimensional network, it validates Equation 10 and allows the expected area of a single contact for fibres of finite length to be determined using Equation 21; this value always being a multiple of $\omega^{2}$ less than $\pi / 2$ since crossings overlapping fibre ends will have area less than $\omega^{2} / \sin (\theta)$. 


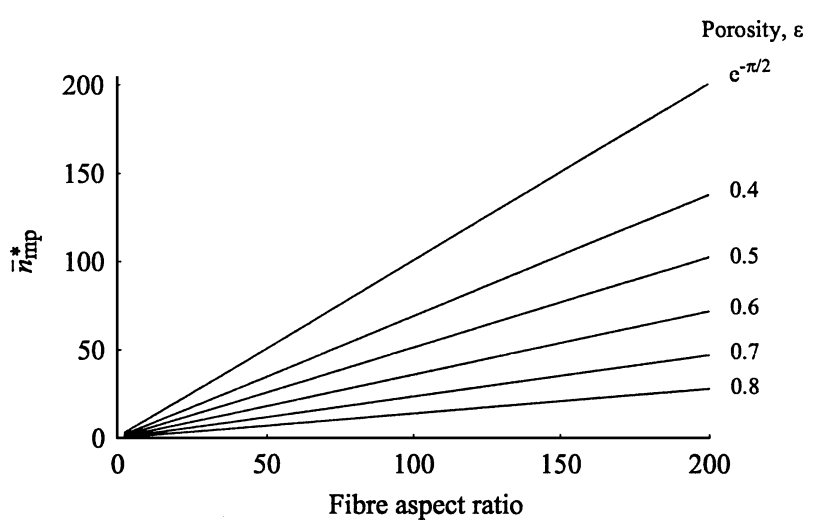

Figure 4 Effect of fibre aspect ratio on the expected number of contacts per fibre for networks of mean coverage $\bar{c}_{\mathrm{mp}}=10$.

Now, Equation 21 gives us the expected area of one contact and Equation 16 gives the fractional contact area of fibres in a multiplanar network considering the contribution of fibres close to the network surface. It follows then that the expected number of contacts per fibre, $\bar{n}_{\mathrm{mp}}^{*}$ is given by,

$$
\begin{aligned}
\bar{n}_{\mathrm{mp}}^{*} & =\frac{2 \lambda \omega}{\bar{A}_{1}} \Phi_{\mathrm{mp}}^{*} \\
& =\frac{\Phi_{\mathrm{mp}}^{*}}{\Phi_{2 \mathrm{D}}^{\text {crit }}}
\end{aligned}
$$

The aspect ratio of a fibre is given by the ratio of its length to its width. The expected number of contacts per fibre, as given by Equation 23, is plotted against fibre aspect ratio in Fig. 4 for networks of mean coverage, $\bar{c}_{\mathrm{mp}}=10$; the number of layers for each value of porosity considered being determined by Equation 14 . In line with expectation, we observe an almost linear increase in the expected number of contacts per fibre with increasing aspect ratio; the gradient of the relationship decreasing with increasing porosity. Taking the limit of $\bar{n}_{\mathrm{mp}}^{*}$ as $\lambda$ and $\bar{c}_{\mathrm{mp}}$ tend to infinity, we find that the maximum expected number of contacts per fibre is $4 / \pi \approx 1.27$ times the aspect ratio. This seems reasonable since the maximum possible number of crossings is twice the aspect ratio and occurs when fibres lie perpendicular to each other with each crossing having area $\omega^{2}$

The expected number of contacts per fibre is plotted against mean network coverage in Fig. 5 for fibres of aspect ratio 100. The broken horizontal lines represent the expected number of contacts per fibre neglecting the contribution of surfaces and calculated by substituting $\Phi_{\mathrm{mp}}$ for $\Phi_{\mathrm{mp}}^{*}$ in Equation 23; the dotted line represents the coverage at which Equation 23 gives $95 \%$ of the expected number of contacts predicted neglecting the contribution of surfaces. As the expected number of contacts per fibre is proportional to the fractional contact area, we observe the same influence of fibres close to the surface as shown in Fig. 3, i.e., the contribution of fibres close to the surface of the network is greater for networks with low porosity than for networks with more open structures.

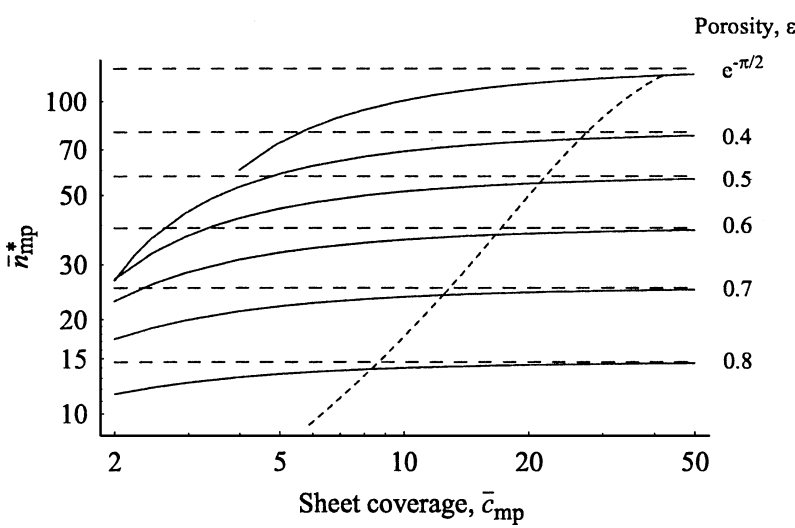

Figure 5 Effect of mean network coverage on the expected number of contacts per fibre for fibres of aspect ratio 100. The broken horizontal lines represent the expected number of contacts per fibre neglecting the contribution of surfaces and the dotted line represents the coverage at which Equation 23 gives $95 \%$ of the expected number of contacts predicted neglecting the contribution of surfaces.

\section{Application and validation}

The amount of contact between fibres determines the available area for inter-fibre bonding in industrially manufactured fibrous networks such as nonwoven fabrics, fibrous filter media and paper. In such materials, the degree of bonding determines their mechanical properties and hence their suitability for given applications. The fractional contact area in such networks is rather difficult to measure experimentally, though the relationship between the strength and optical properties of paper formed from natural fibres has been shown by Ingmanson and Thode [15] to provide an estimate of the relative bonded area. The technique uses measurements of the specific light scattering coefficient, $s$ $\left(\mathrm{m}^{2} \mathrm{~g}^{-1}\right)$ and tensile strength of samples made from a given fibre type such that,

$$
\mathrm{RBA}=1-\frac{s}{s_{0}},
$$

where $s_{0}$ is the specific light scattering coefficient of an unbonded sheet and is determined by extrapolation of a plot of light scattering coefficient against tensile strength. Inherent in the method are the assumptions that in regions where fibres are in mutual optical contact, they are bonded also and that the extrapolation to determine $s_{0}$ is reliable. This second assumption is important. In a study of papers formed from various wood pulp fibres, Rennel [16] with Hartler [17] investigated the relationships among density, light scattering coefficient, tensile strength and the surface area of fibres as determined by nitrogen adsorption for freezedried, solvent exchange dried and spray dried fibres. Of particular relevance to the work presented here is the conclusion drawn by Rennel and Hartler that there is no reason to suppose that a unique relationship exists between fibre surface area and the light scattering coefficient. Typically, we expect the Relative Bonded Area obtained using the approach of Ingmanson and Thode [15] to provide an overestimate; nevertheless, data obtained using this technique are widely available in the literature and represent the best available experimental evidence against which the model may be tested. Note 
also that the fractional contact area, for which we have derived expressions here, represents an upper bound on the relative bonded area and is equivalent to the relative bonded area only when all areas of contact are bonded.

The general literature provides some data for both the porosity or density, and the fractional contact area. Yang et al. [18] measured the regions of contact of micrographs cross-sections of three paper samples manufactured on papermaking machines. Jowsey [19] presents data for the relative bonded area and density of laboratory formed fibre networks pressed to differing degrees and treated with starch to improve inter-fibre bonding; Moss [20] gives similar data for laboratory formed networks made from three fibre types each subjected to mechanical work; Wilde [21] gives data for networks formed on a pilot scale papermaking machine from blends of two fibre types subjected to different mechanical treatments. Neither Moss nor Wilde report the relative bonded area, but both report measurements of tensile strength, optical properties and density. For comparison with the model presented here, their data have been processed to yield the relative bonded area using Equation 24. Note also that whilst the structure of the laboratory formed networks of Jowsey and Moss may be considered close to random, the structure of the machine made networks of Wilde can be expected to exhibit significant departures from randomness due to interaction between fibres and hydrodynamic effects; for discussion of these see Sampson [22].

The relative bonded area is plotted against porosity for the data of Jowsey, Moss, Wilde and Yang et al. in Fig. 6. Since the coverage of the networks was not reported, the number of layers constituting the networks cannot be calculated; accordingly the data are compared with the fractional contact area calculated without accounting for surface effects using Equation 13. Porosities have been estimated from the reported densities of networks using,

$$
\varepsilon=1-\frac{\rho_{\text {network }}}{\rho_{\text {fibre }}}
$$

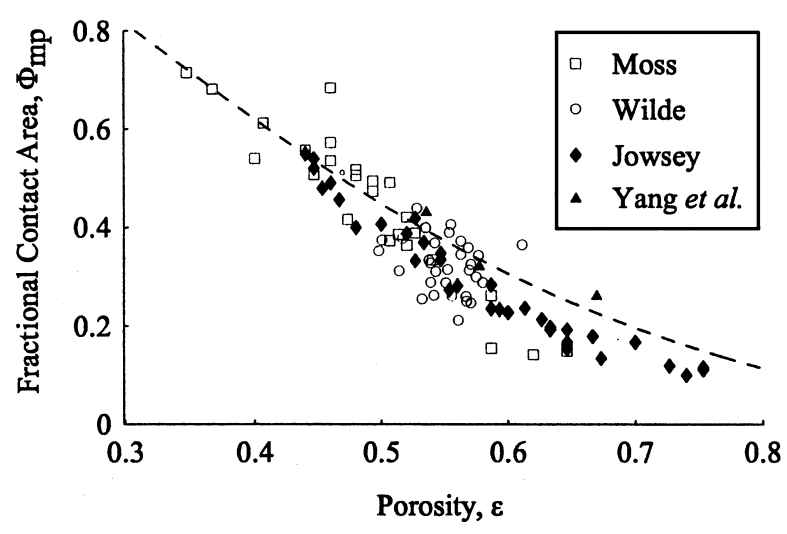

Figure 6 Comparison of experimental data for Relative Bonded Area with Equation 13 for the fractional contact area of a fibre in the bulk of multiplanar network. where $\rho_{\text {network }}$ and $\rho_{\text {fibre }}$ are the densities of the networks and the fibres respectively; the density of fibres has been assumed to be that of cellulose, the main constituent of wood pulp fibres, i.e., $\rho_{\text {fibre }}=1.5 \mathrm{~g} \mathrm{~cm}^{-3}$. From the discussion of Fig. 3 we expect the fractional contact area as predicted by Equation 13 to be greater than that given by Equation 16 because fibres close to the surfaces of the network are in contact with fewer other fibres than those in the bulk of the network. Despite this, the agreement between theory and experiment shown in Fig. 6 is good, particularly considering the different sources of data, fibre types, fibre treatments, the nonrandom nature of the machine-made samples and the assumption of constant fibre density for all samples.

Data for the fractional contact area measured from micrographs of laboratory formed networks are given by Paavilainen [23] and Niskanen and Rajatora [11]. As well as measuring the fractional contact area, both articles report mean coverage allowing validation of Equation 16 that accounts for surface effects. Niskanen and Rajatora report network porosity also, whilst Paavilainen reports network thickness and assumed the mass per unit area of all networks to be $60 \mathrm{gm}^{-2}$ allowing porosity to be estimated, again assuming the density of fibres to be that of cellulose. Niskanen and Rajatora note also that the 'bonding ratio' reported by Paavilainen is, in fact $\Phi_{\mathrm{mp}}^{*}-1 / \bar{c}$ and accordingly the data have been corrected for use here.

The fractional contact area calculated using Equation 16 with the coverage and porosity data of Paavilainen and Niskanen and Rajatora is plotted against the measured fractional contact area in Fig. 7 where the broken line has unit gradient. Whilst agreement with the data of Paavilainen is good, the model yields estimates of the fractional contact area that are more than double the values reported by Niskanen and Rajatora. The precise cause of the discrepancy between the model and the data of Niskanen and Rajatora is unclear, though in the discussion of their data, they noted that their estimates of fractional contact area were lower

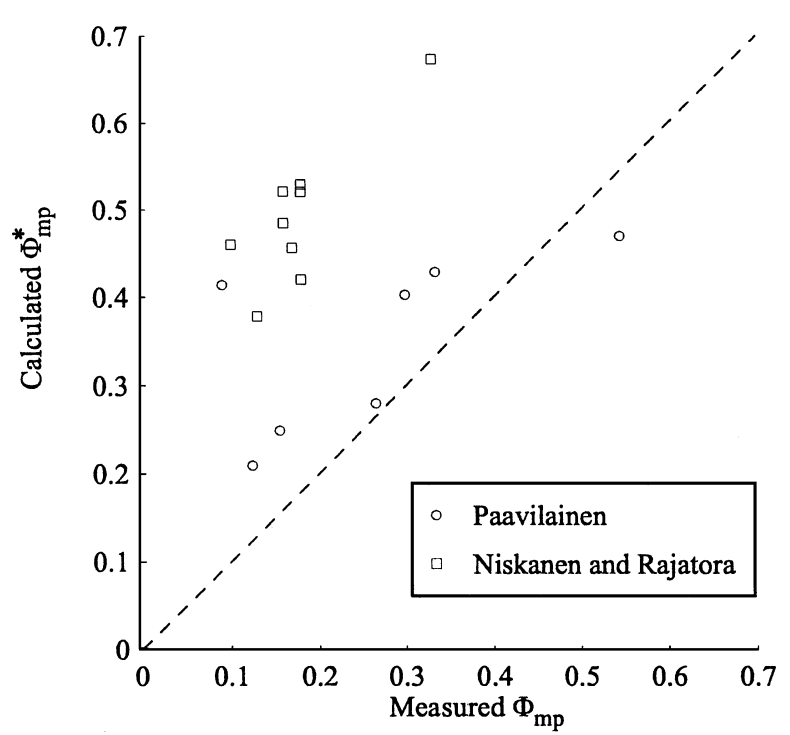

Figure 7 Comparison of fractional contact area calculated considering surface contribution with data of Paavilainen [23] and Niskanen and Rajatora [11]. 
than those of Yang et al. [18] and Paavilainen [23] who used similar measurement techniques on samples of similar density. We note that the data of Yang et al. and those of Paavilainen yield estimates of the fractional contact area much closer to those obtained by Jowsey, Moss and Wilde using optical techniques.

\section{Discussion}

The theory presented here allows the fractional contact area in random fibrous networks to be calculated from the expected coverage of the network and the network porosity. For sufficiently thick networks, knowledge of the porosity only is required. Previous models are either applicable only to thin networks that are not representative of industrial formed structures $[5,7]$, or require knowledge of either fibre thickness $[10,13]$ or parameters associated with the measurement technique [11]. Here, the coverage of a layer has been defined in terms of network porosity only, this being an intrinsic property of the structure. Determination of the mean coverage of multiplanar structures requires knowledge of the width and coarseness of the fibres and these parameters are much easier to determine than fibre thickness as required by the models given in the literature. The model allows also determination of the expected area of a single crossing and hence of the expected number of crossings per fibre.

The theory has been compared with experimental data for networks of porosity of above 0.3 and a fractional contact area of about 0.75 . Whilst the expressions are valid for porosities above, corresponding to a porosity of around 0.21 , this lower limit yields a prediction of fractional contact area approaching 1. Clearly this condition can only be met by having a porosity of zero. The theory presented here applies strictly to projections of the fibre surface in the $x-y$ plane. As such, the predictions given by the theory are probably most reliable for networks of porosity greater than about 0.3 .

\section{Conclusions}

Expressions have been derived for the fractional contact area in random fibre networks. Three cases have been considered: two dimensional networks and multiplanar networks formed from the superposition of two-dimensional networks neglecting and including the contribution of surfaces. In the first two cases, the fractional contact area may be expressed in terms of the porosity only; in the third case the expected coverage of the network must be considered also. The expressions derived have been compared with experimental data from the literature and the agreement is good. Given the fractional contact area, an estimate of the expected number of contacts per fibre can be made; the quality of this estimate is difficult to test, though its dependence on network porosity and fibre aspect ratio seems appropriate.

\section{References}

1. R. E. MILES, Proc. Nat. Acad. Sci. USA 52 (1964) 901, 1157.

2. H. W. PIEKAAR and L. A. ClARENB URG, Chem. Eng. Sci. 22 (1967) 1399.

3. H. CORTE and E. H. LLOYD, in "Consolidation of the Paper Web,” Trans. IIIrd Fund. Res. Symp., edited by F. Bolam (BPBMA, London, 1966) p. 189. See also Discussion following, p. 1010.

4. C. T. J. DODSON and W. W. SAMPSON, Appl. Math. Lett. 10(2) (1997) 87.

5. O. KALLMES and H. CORTE, Tappi J. 43(9) (1960) 73; Errata. 44(6) (1961) 448.

6. C. T. J. DOdSON, J. Roy. Statist. Soc. B 33(1) (1971) 88.

7. M. DENG and C. T. J. DODSON, "Paper: An Engineered Stochastic Structure" (Tappi Press, Atlanta, 1994).

8. O. KALlmeS, H. CORTE and G. BERNiER, Tappi J. 46(8) (1963) 493.

9. B. RADVAN, C. T. J. DODSON and C. G. SKOLD, in "Consolidation of the Paper Web," Trans. IIIrd Fund. Res. Symp., edited by F. Bolam (BPBMA, London, 1966) p. 189.

10. R. M. SOSZYŃS KI, Nord. Pulp Pap. Res. J. 10(2) (1995) 150.

11. K. NiSKANEN and H. RAJATORA, J. Pulp Pap. Sci. 28(7) (2002) 228.

12. E. K. O. HELLÉN, M. J. ALAVA and K. J. NISKANEN, J. Appl. Phys. 81(9) (1997) 6425.

13. D. J. GATES and M. WES TCOT T, J. Stat. Phys. 94(1/2) (1999).

14. W. W. SAMPSON, J. Mater. Sci. 38(8) (2003) 1617.

15. W. L. INGMANSON and E. F. THODE, Tappi J. 42(1) (1959) 83.

16. J. RENNEL, Svensk Papperstidn. 72(1) (1969) 1.

17. N. HARTLER and J. RENNEL, ibid. 72(1) (1969) 9.

18. C.-F. YANG, A. R. K. EUSUFZAI, R. SANKAR, R. E. MARK and R. W. PERKINS, ibid. 81(13) (1978) 426.

19. C. J. JOWSEY, M.Sc. Thesis, Department of Paper Science, UMIST, 1984

20. P. A. MOS S, Ph.D. Thesis, Department of Paper Science, UMIST, 1992.

21. R. WIL DE, M.Phil. Thesis, Department of Paper Science, UMIST, 2000 .

22. W. W. SAMPSON, in "The Science of Papermaking," Trans. XIIth Fund. Res. Symp., edited by C.F. Baker (Pulp and Paper Fundamental Research Society, Bury, 2001) p. 1205.

23. L. PAA Vilainen, Pap. ja Puu 76(3) (1994) 162.

Received 10 April 2003

and accepted 12 January 2004 